\title{
Brain electrical signal pre-amplification system design
}

\author{
ZHUANG Qiu hui ${ }^{1,}$ a , WANG San qiang ${ }^{2, b}$, XING LIANG $^{3, b}$ \\ ${ }^{1}$ Chongqing University of Technology, Chongqing 400054,China \\ ${ }^{2,3}$ State Grid Chongqing Electric Power CO. Electric Power Research Institute, Chongqing \\ 401123, China \\ azqh@cqut.edu.cn, bwsqcqu@163.com \\ Fund project: the natural science foundation of China \\ Item number: 51275551 \\ Fund project: the natural science foundation of China \\ Item number: 51505053
}

Keywords: brain electrical signal pre-amplification signal processing

Abstract: EEG contains a wealth of physiological, psychological and pathological information, EEG signal analysis and processing are either in clinical of some brain disease diagnosis and treatment, or in the cognitive science research fields are very important. According to the basic characteristics of EEG signal, discusses the EEG signal acquisition principle, method and the design train of thought. Proposed pre-amplification system design, after prestage processing of EEG signals, can be seen in the EEG signals transform trend, coupled with the follow-up processing, can get the clear stability of EEG signal, and has good application value.

\section{Introduction}

In the cerebral cortex, there are frequent electrical activity, this activity is spontaneous, and it is through these people electrical activity to complete various physiological function. The electrical activity of the potential change over time, electrode the potential time-varying waveforms extracted and recorded by EEG. Brain electrical signal is the cerebral cortex or scalp surface recording to the cerebral cortex neuron postsynaptic potential is the sum of group ${ }^{[1]}$. EEG recording of spontaneous electrical activity of the brain is the human, this spontaneous EEG in clinical diagnosis has important significance. The detection and recording of the EEG can be on the human brain and nervous system disease diagnosis and treatment, so it is very necessary to study the eeg. Modern medical science has established a normal EEG diagnostic criteria and abnormal EEG diagnostic criteria, therefore, EEG in clinical diagnosis has the extremely important value ${ }^{[2]}$. Medical EEG examination can assist physicians for medical assessment, diagnosis and monitoring of medical process, including the application of epilepsy, Alzheimer's disease, encep- halitis, brain tumor, brain injury and brain function decline in various brain diseases. In addition, this technique can also be applied to human sleep research and nerve relaxation training, Expanding the EEG detection and analysis technology of range ${ }^{[3]}$ 。

\section{system design}

EEG signal processing circuit is the task of extracting eeg signal from noise, and enlarge it to the appropriate level.

In EEG signal processing, pre-amplification circuit design is the key link of pre-amplification, only to achieve success, it can ensure the follow-up work further. Pre stage connected into a differential amplifier form has two functions, namely the amplified differential signal (EEG), 
suppression of common mode signal (interference).Basic differential amplifying circuit has input resistance high enough problems, because the differential input voltage from the amplifier non-inverting terminal and the inverting on both sides at the same time to join. If the differential input signal from the in-phase side into, can greatly improve the input impedance of the circuit, namely parallel connected noninverting differential amplifying circuit. As shown in Figure 3.1, the input impedance can be up to above 10M. Figure A1, A2 consisting of parallel connected noninverting input first stage amplifier, to increase the input impedance of the amplifier.A3 differential amplifier, the amplifier second.

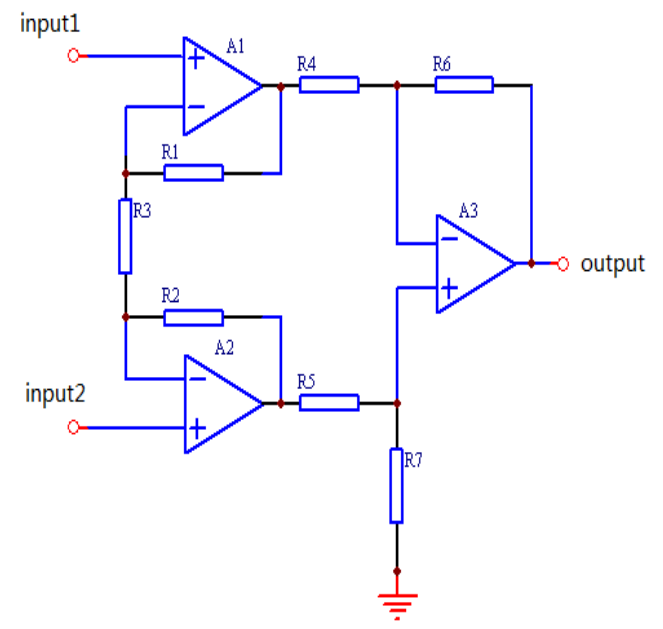

Fig 1 Inphase-paralleled differential amplifier circuit

Using parallel connected noninverting differential amplifier circuit, the designed circuit as shown in figure2.The circuit of the traditional phase parallel pre-amplification circuit made improvements, in the first stage amplifying circuit followed by two capacitors, used to remove the polarization voltage.

A,B consisting of parallel connected noninverting input first stage amplifier, to increase the input impedance of the amplifier.U2A differential amplifier, the amplifier second.

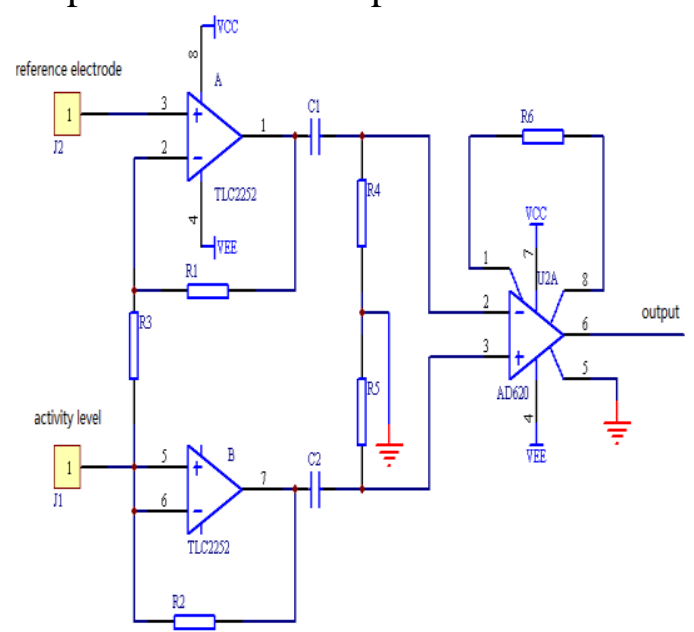

Fig 2 Frontal Magnify Circuit

The first stage amplifier circuit using TLC2252, TLC2252 has a high input impedance and a low noise characteristic, can be well applied to high impedance source, its low power consumption, but also has low input bias current and low input offset voltage characteristics, suitable for the design of this issue.

The first level voltage gain:

$$
A_{d 1}=1+\frac{R_{1}+R_{2}}{R_{3}}
$$


A preamplifier circuit of grade second, using a new type of high performance instrumentation amplifier AD620 as EEG preamplifier core device.Using AD620 as the second level voltage gain: $A_{d 2}=1+\frac{49.4 k}{R_{6}}$

The prestage voltage gain is bigger, behind all the circuits caused by the influence of noise is small, therefore, the prestage gain should be as high as possible. But the prestage voltage gain by the amplifier dynamic range limits, and in the removal of the electrode polarization voltage before the prestage, dynamic range by the polarization voltage limits. The front stage circuit is divided into two stages of amplification. The prestage, first level voltage gain, the amplifier noise impact is the biggest, the first level high voltage gain, will improve the signal quality, high signal to noise ratio.

The total gain of the amplifying circuit:

$G=A_{d 1} \times A_{d 2}=\left(1+\frac{R_{1}+R_{2}}{R_{3}}\right)\left(1+\frac{49.4 k}{R_{6}}\right)$

The prestage circuit for two stage amplifier, you can modify the $R_{1}\left(R_{2}\right), R_{3}$ and $R_{6}$ to change the magnification, prestage circuit magnification must be as large but not damage signal linearity, proved by the experiment, take 2000 times magnification is reasonable. The specific $R_{1}\left(R_{2}\right), R_{3}$ and $R_{6}$ value can be determined by experiment: respectively the circuit of Figure $3.2 R_{1}\left(R_{2}\right)=50 \mathrm{~K} \Omega, \quad R_{3}=1 \mathrm{~K} \Omega, \quad R_{6}=2.5 \mathrm{~K} \Omega$ namely pre-amplification circuit two stage amplifying set respectively 100 times, 20 times. Because the system total magnification of 15000 times, the last stage of amplification can be made adjustable gain, a magnification of 8 times or so can meet the requirements.

Using Figure 2 circuit, through the brain electrode for the detection of brain waves, observation oscilloscope output as shown in figure 3.

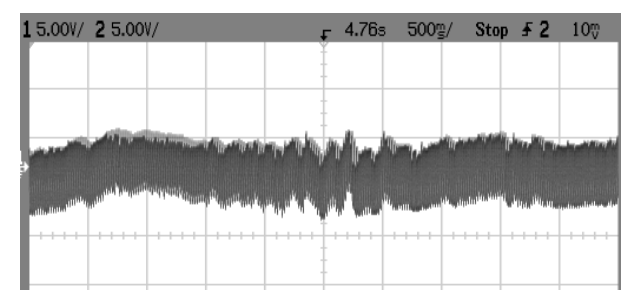

Fig 3 Output voltage waveform of preamplifier

From the graph 3 can see, brain waves remains submerged in the $50 \mathrm{~Hz}$ interference, but has to be seen in brain wave change trend. In the pre-amplification circuit followed by a superior $50 \mathrm{~Hz}$ notch filter, oscilloscope is as shown in Figure 4 EEG waveform.

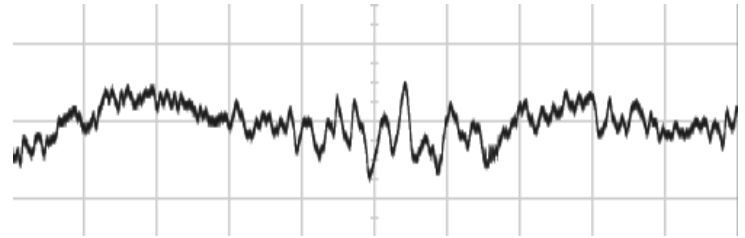

Fig 4 Output EEG waveform of $50 \mathrm{~Hz}$ notch filter

\section{Conclusions}

Experiment we used this circuit for EEG signal pre-amplification, the effect is ideal, although still need to filter circuit to eliminate power-line interference, but compared to conventional EEG detection circuit, the circuit is simplified a lot, it helps to design a multi channel EEG machine has the practical significance. At the same time, because the circuit in the front level namely the 
amplification of several thousands of times, so that brain wave shape maximum distortion. Of course, we can see from Figure 4, the way to get an EEG waveform baseline drift is relatively large, also need to be at the back of the circuit to suppress. In general, the present system for EEG signal pre-amplification circuit has the advantages of simple structure, convenient debugging, good effect, for the EEG amplifier and other bioelectric amplifier provides a new train of thought.

\section{Acknowledgements}

This work was financially supported by The Chongqing Natural Science Fund Projects (cstc2012jjA40062).

\section{References}

[1] Yu Xuefei, Principle and design of medical electronic equipments, South China University of Technology press, .102-162,(2000)

[2] Deng Qinkai, Principle of modern medical instrumentation design, Science Press, 20-23(2004)

[3] Wolpaw J R ,Birbaumer N,Heetderks WJ,et al.Brain-computer interface technology:A review of the first international meeting IEEE Trans Rehab Eng, 8(2):164(2000)

[4] Huang Yuangui, Wu Shengling, etc. Clinical electroencephalography, Shaanxi science and Technology Press(1984)

[5] Yang Wenxiu Li Zhengming, Biomedical Physics(1993)

[6] Huang Zhiwei, Digital wireless transceiver circuit design, Publishing House of electronics industry(2003)

[7] Feng Yingkun, Clinical medical science, people's Medical Publishing House(1984)

[8]Biocybernaut Institute, "EEG- ElectroEncephaloGraph", 2000-2002 Biocybernant Institute Mountain View, Califonia

[9] Thomas F. Collura, The Application of Small brainwave machine, http://www.brainmaster.com,2001

[10]Huan fei,Wang Zhi Zhong ,ChongXun “Analysis of Sleep EEG by Using Multi-Scale Entropy”, Beijing Journal of Biomedical Engineering(2001)

Author brief introduction:

Zhuang Qiu hui (1979-), female, lecturer at the Chongqing University of Science and Technology, engaged in electronic technology research. 